\title{
Development of a Novel Chimeric Endolysin, Lys109 With Enhanced Lytic Activity Against Staphylococcus aureus
}

OPEN ACCESS

Edited by:

Naomi Sulinger Hoyle,

Eliava Phage Therapy Center (EPTC),

Georgia

Reviewed by:

Hidetomo Iwano

Rakuno Gakuen University, Japan Malgorzata Barbara Lobocka,

Institute of Biochemistry

and Biophysics (PAN), Poland

*Correspondence:

Sangryeol Ryu

sangryu@snu.ac.kr

Specialty section:

This article was submitted to

Antimicrobials, Resistance

and Chemotherapy,

a section of the journa

Frontiers in Microbiology

Received: 10 October 2020

Accepted: 16 December 2020

Published: 15 January 2021

Citation:

Son $B$, Kong $M$, Lee $Y$ and Ryu S

(2021) Development of a Novel

Chimeric Endolysin, Lys109 With

Enhanced Lytic Activity Against

Staphylococcus aureus.

Front. Microbiol. 11:615887.

doi: 10.3389/fmicb.2020.615887

\section{Bokyung Son ${ }^{1,2}$, Minsuk Kong ${ }^{3}$, Yoona Lee ${ }^{1,2}$ and Sangryeol Ryu ${ }^{1,2,4 *}$}

${ }^{1}$ Department of Food and Animal Biotechnology, Seoul National University, Seoul, South Korea, ${ }^{2}$ Department of Agricultural Biotechnology, Seoul National University, Seoul, South Korea, ${ }^{3}$ Department of Food Science and Technology, Seoul National University of Science and Technology, Seoul, South Korea, ${ }^{4}$ Center for Food and Bioconvergence, Research Institute of Agriculture and Life Sciences, Seoul National University, Seoul, South Korea

As the incidence of antibiotic-resistant bacteria has become increased, phage endolysins are believed as one of the promising alternatives to antibiotics. However, the discovery of potent endolysin is still challenging because it is labor intensive and difficult to obtain a soluble form with high lytic activity. In this respect, the modular structures of Gram-positive endolysins can provide an opportunity to develop novel endolysins by domain rearrangement. In this study, a random domain swapping library of four different endolysins from phages infecting Staphylococcus aureus was constructed and screened to obtain engineered endolysins. The novel chimeric endolysin, Lys109 was selected and characterized for its staphylolytic activity. Lys 109 exhibited greater bacterial cell lytic activity than its parental endolysins against staphylococcal planktonic cells and biofilms, showing highly improved activity in eliminating S. aureus from milk and on the surface of stainless steel. These results demonstrate that a novel chimeric endolysin with higher activity and solubility can be developed by random domain swapping and that this chimeric endolysin has a great potential as an antimicrobial agent.

Keywords: Staphylococcus aureus, endolysin, domain swapping, screening, antimicrobial agent

\section{INTRODUCTION}

Staphylococcus aureus is a Gram-positive bacterium that threatens human and animal health, causing staphylococcal food poisoning and a wide range of infectious diseases, including skin infections, pneumonia, meningitis, endocarditis, and osteomyelitis (Lowy, 1998; De Lencastre et al., 2007). In particular, the global spread of methicillin-resistant S. aureus (MRSA) has raised serious concerns because MRSA can easily become resistant to multiple antibiotics, limiting treatment options (Chambers and Deleo, 2009). Moreover, the strong biofilm-forming ability of S. aureus has aggravated problems in the food and medical industries (Lewis, 2001; Otto, 2012). For these reasons, there is an urgent need to create new antimicrobials to combat S. aureus (Foster, 2004).

Endolysins are bacteriophage-encoded peptidoglycan hydrolases produced by bacteriophages at the end of their replication cycle to breakdown peptidoglycans of the bacterial cell wall, resulting in the release of viral progeny (Schmelcher et al., 2012a). Endolysins have been suggested as promising 
antibacterial agents because purified endolysin proteins can rapidly lyse and induce death in Gram-positive bacteria when applied exogenously. Compared to classical antibiotics, endolysins have several advantages because they have narrow host specificity, high sensitivity, and a low probability to develop bacterial resistance (Borysowski et al., 2006). Gram-positive endolysins have a modular architecture with at least two separate functional regions. Generally, the N-terminal domain carries more than one catalytic domain and is attached to the C-terminal cell wall-binding domain (CBD) by a short linker (Fischetti, 2008; Schmelcher et al., 2012a). The catalytic domain determines the enzymatic activity of the endolysin, whereas the CBD positions the catalytic domain to the peptidoglycan of the target bacteria for efficient lysis by the endolysin (Loessner, 2005; Schmelcher et al., 2011).

Most endolysins of staphylococcal phages have three distinct domains: an N-terminal cysteine- and histidinedependent amidohydrolase/peptidase (CHAP) domain, a central N-acetylmuramoyl-1-alanine amidase (Ami_2 or Ami_3) domain, and a C-terminal SH3b domain as a CBD (Chang and Ryu, 2017). The efficacy of S. aureus phage endolysins killing $S$. aureus and controlling staphylococcal infection in animal models has been reported in several studies (Kerr et al., 2001; Rashel et al., 2007; Fenton et al., 2010; Gu et al., 2011). Although several $S$. aureus phage endolysins have presented promising results, poor expression levels and/or insolubility of the expressed proteins have limited the development of highly active staphylococcus-specific phage endolysins (Daniel et al., 2010). In addition, identifying a novel endolysin from $S$. aureus phages is relatively difficult because most $S$. aureus-targeting endolysins have similar domain compositions and display high amino acid sequence identity (Becker et al., 2009b; Oliveira et al., 2013; Chang and Ryu, 2017). To circumvent these problems, a number of research groups have designed truncated or chimeric versions of lysins (Manoharadas et al., 2009; Idelevich et al., 2011; Fernandes et al., 2012), but these trial-and-error strategies are time-consuming and labor-intensive in the search to find a novel endolysin with the desired properties. The modular structure of the functional domains of Gram-positive endolysin allows us to engineer endolysins through domain swapping to generate chimeric endolysins with superior properties (Diaz et al., 1990; Schmelcher et al., 2010). Endolysin engineering such as rationally designed domain recombination or random domain swapping has been endeavored. For example, Yang et al. reported an improved screening of a random domain recombination library of endolysins using controlled lysis of E. coli (Yang et al., 2015).

In this study, we developed an induced lysis-based screening to improve the screening efficiency further, enabling us to identify nineteen new chimeras containing different combinations of catalytic and cell wall binding domains from four S. aureus phage endolysins. Among them, a novel chimeric endolysin, Lys109, which showed enhanced lytic activity against $S$. aureus and other multiple staphylococcal species, was selected and characterized. This proof-of-concept study confirms the potential of the random domain swapping method to develop a novel therapeutic agent to control S. aureus.

\section{MATERIALS AND METHODS}

\section{Bacterial Strains and Growth Conditions}

The bacterial strains used in this study are listed in Table $\mathbf{1 .}$ Staphylococcal strains were grown in tryptic soy broth (TSB) (Difco, Detroit, MI, United States) at $37^{\circ} \mathrm{C}$ under aerobic conditions. Baird-Parker agar plates with egg yolk tellurite (BPA; Difco) were used for the selective enumeration of S. aureus. Bacillus cereus, B. subtilis, Listeria monocytogenes, and Streptococcus thermophilus was cultivated in brain heart infusion (BHI) medium (Difco). Luria-Bertani (LB) medium (Difco) was used for the growth of Gram-negative strains. Escherichia coli $\mathrm{DH} 5 \alpha$ and BL21 (DE3) star strains were used to clone and express proteins, respectively.

\section{Library Construction for Random Domain Swapping}

The gene encoding SPN1S lysRz (SPN1S_0028 and SPN1S_0029) was amplified from Salmonella Typhimurium phage SPN1S (GenBank accession number NC_016761) using the oligonucleotides listed in Supplementary Table S1. The gene fragment was digested with EcoRI-SalI and inserted into the pBAD33 vector ( $\operatorname{Lim}$ et al., 2012). To confirm the cell lysis efficiency, the pET28a_EGFP vector (Kong and Ryu, 2015) was cotransformed into competent E. coli BL21 (DE3) with pBAD33_SPN1S lysRz. The fluorescence of the released EGFP was measured by using a SpectraMax i3 multimode microplate reader (Molecular Devices, Sunnyvale, CA, United States) with excitation at $485 \mathrm{~nm}$ and emission at $535 \mathrm{~nm}$. Four different S. aureus phage endolysins, including LysSA12, LysSA97, LysSA11, and LysSAP4, were used to construct two types of libraries (Chang and Ryu, 2017; Chang et al., 2017a). For the first library, we tried to generate chimeric endolysins containing two catalytic domains. The genes encoding four CHAP domains and those encoding three amidase domains from the four endolysins were amplified and digested with BamHI/XhoI for insertion as the N-terminal domain of the chimeric endolysins. For the central domain of chimeric endolysins, the seven genes were amplified and digested with XhoI/BamHI. All plasmids and primers used in this study are listed in Supplementary Table S1. For the second library, all genes encoding CHAPs and amidase domains were amplified and digested with only BamHI to ensure diversity in the number of catalytic domains to be inserted into the endolysins. All gene fragments were randomly ligated into the BamHI sites of pET28a vectors (Novagen, Madison, WI, United States) containing one of the genes encoding four different cell wall binding domains from the four endolysins. The vector libraries were cotransformed into competent E. coli BL21 (DE3) harboring pBAD33_SPN1S lysRz.

\section{Screening of the Chimeric Endolysins by the Plate Lysis Method}

The resulting clones from the random library were screened as described in a previous study with some modifications (Yang et al., 2015). In brief, the clones were picked and grown in 96well plates with fresh LB broth containing $0.01 \mathrm{mM}$ Isopropyl 
TABLE 1 | Antimicrobial spectrum of LysSA12 and Lys109.

\begin{tabular}{|c|c|c|c|c|c|}
\hline \multirow[t]{3}{*}{ Species } & \multirow[t]{3}{*}{ Strains } & \multicolumn{4}{|c|}{ Antimicrobial activity ${ }^{a}$} \\
\hline & & \multicolumn{2}{|c|}{ LysSA12 (pmol) } & \multicolumn{2}{|c|}{ Lys109 (pmol) } \\
\hline & & 167 & 16.7 & 167 & 16.7 \\
\hline \multirow[t]{19}{*}{ S. aureus } & Human isolate 117 & + & - & ++ & + \\
\hline & Human isolate 119 & + & - & ++ & - \\
\hline & Plant isolate 37 & + & - & ++ & - \\
\hline & Plant isolate 8 & + & - & ++ & - \\
\hline & Plant isolate 13 & + & - & + & - \\
\hline & Animal isolate 154 & + & - & ++ & - \\
\hline & Animal isolate 134 & + & - & ++ & - \\
\hline & Animal isolate 100 & + & - & ++ & - \\
\hline & Animal isolate 99 & + & - & ++ & - \\
\hline & Clinical isolate 1163 & + & - & ++ & - \\
\hline & Clinical isolate FMB1 & + & - & ++ & - \\
\hline & Clinical isolate FMB2 & + & - & ++ & - \\
\hline & Clinical isolate FMB3 & + & - & ++ & - \\
\hline & Mastitis cow milk isolate FMB4 & ++ & - & ++ & + \\
\hline & ATCC 6538 & + & - & ++ & - \\
\hline & $\mathrm{RN} 4220$ & + & - & ++ & - \\
\hline & ATCC 23235 & + & - & ++ & + \\
\hline & ATCC 13301 & + & - & ++ & - \\
\hline & CCARM 3090 & + & - & ++ & - \\
\hline S. hominis & ATCC 37844 & + & - & ++ & + \\
\hline S. saprophyticus & ATCC 15305 & + & - & ++ & + \\
\hline S. haemolyticus & ATCC 29970 & + & - & ++ & - \\
\hline S. capitis & ATCC 35661 & + & - & ++ & - \\
\hline S. warneri & ATCC 10209 & + & - & + & - \\
\hline S. xylosus & ATCC29971 & + & - & + & - \\
\hline S. epidermidis & CCARM 3787 & ++ & + & ++ & ++ \\
\hline Bacillus cereus & KCCM 40133 & - & - & - & - \\
\hline B. subtilis & 168 & - & - & - & - \\
\hline Streptococcus thermophilus & ATCC 19258 & - & - & - & - \\
\hline Listeria monocytogenes & ATCC 19114 & - & - & - & - \\
\hline Salmonella Typhimurium & LT2 & - & - & - & - \\
\hline Pseudomonas aeruginosa & ATCC 27853 & - & - & - & - \\
\hline Cronobacter sakazakii & ATCC 29544 & - & - & - & - \\
\hline Escherichia coli & MG1655 & - & - & - & - \\
\hline
\end{tabular}

a ++ clear lysis zone, + turbid lysis zone, - no lysis zone.

$\beta$-D-1-thiogalactopyranoside (IPTG) and grown overnight at $37^{\circ} \mathrm{C}$ to initiate the expression of the chimeric endolysins. Afterward, $0.2 \%$ arabinose was added to the wells to express SPN1S lysRz. These cultures were dotted onto agar plates overlaid with autoclaved S. aureus RN 4220 and incubated for $12 \mathrm{~h}$ at $37^{\circ} \mathrm{C}$. The clones exhibiting a clear lysis zone against $S$. aureus were screened and picked for sequencing analysis to identify the cloned chimeric endolysin.

\section{Expression and Purification of the Endolysins}

Escherichia coli BL21 (DE3) harboring chimeric endolysins were cultivated at $37^{\circ} \mathrm{C}$ to an optical density at $600 \mathrm{~nm}\left(\mathrm{OD}_{600}\right)$ of 0.7 and the protein expression was induced by addition of $0.5 \mathrm{mM}$ IPTG for $20 \mathrm{~h}$ at $18^{\circ} \mathrm{C}$. Bacterial cells were suspended in lysis buffer $(50 \mathrm{mM}$ sodium phosphate, $300 \mathrm{mM}$ sodium chloride and $30 \%$ glycerol; $\mathrm{pH} 8.0$ ) and disrupted by sonication at a duty cycle of $25 \%$ and output control of 5 (Branson Ultrasonics, Danbury, CT, United States). After centrifugation $(20,000 \times g$, $30 \mathrm{~min}$ ), the supernatant was passed through a Ni-NTA superflow column (Qiagen GmbH, Hilden, Germany), and purification of the recombinant proteins was performed according to the manufacturer's instructions. The purified protein was stored at $-80^{\circ} \mathrm{C}$ until use after the buffer was changed to storage buffer (50 mM sodium phosphate, $300 \mathrm{mM} \mathrm{NaCl}$ and $30 \%$ glycerol; $\mathrm{pH}$ 8.0) using PD Miditrap G-25 (GE Healthcare, Amersham, Bucks, United Kingdom). 


\section{Lytic Activity Assay}

The lytic activity of the chimeric endolysins and their original endolysins was assessed with a turbidity reduction assay (Son et al., 2012). Bacterial cells grown to the exponential phase were resuspended in reaction buffer ( $50 \mathrm{mM}$ Tris- $\mathrm{HCl}, \mathrm{pH} 6.5$ ). Then, the purified proteins were added to the cell suspension at a final concentration of $300 \mathrm{nM}$, and the OD reduction of the cells was measured over time by using a SpectraMax i3 multimode microplate reader at $600 \mathrm{~nm}$. The relative lytic activity was calculated after $60 \mathrm{~min}$ as follows: $\left[\Delta \mathrm{OD}_{600}\right.$ test (endolysin added) - $\Delta \mathrm{OD}_{600}$ control (buffer only)]/initial $\mathrm{OD}_{600}$. The antimicrobial spectrum was tested by a plate lysis assay as previously described (Chang et al., 2017a). In brief, $10 \mu \mathrm{L}$ of diluted endolysin $(167,16.7$, and $1.67 \mathrm{pmol})$ was spotted onto a freshly prepared bacterial lawn on TSA agar plates. Spotted plates were air-dried in a laminar flow hood for $15 \mathrm{~min}$ and incubated overnight at $37^{\circ} \mathrm{C}$. The MIC of the endolysins was determined by serial dilution of the endolysins by 1:2 in 96well plates as described previously (Andrews, 2001; Swift et al., 2015). Exponentially growing $S$. aureus CCARM 3090 was added to each well at a final concentration of $10^{5} \mathrm{CFU} /$ well, and the plate was incubated at $37^{\circ} \mathrm{C}$ for $20 \mathrm{~h}$. The MIC was defined as the lowest concentration of endolysin that produced inhibition of visible growth.

\section{Biofilm Reduction Assay}

The biofilm reduction assay was performed as previously described with some modifications (Wu et al., 2003). Staphylococcal strains incubated in TSB medium supplemented with $0.25 \%$ D-glucose (Sigma-Aldrich, St. Louis, MO, United States) were prepared and subcultured in the same media in a 96-well polystyrene microplate. After incubating the microplate for $24 \mathrm{~h}$ at $37^{\circ} \mathrm{C}$, all wells were washed with PBS. Once the biofilm formed, the experimental group wells were filled with endolysins. After incubation for $2 \mathrm{~h}$ at $37^{\circ} \mathrm{C}$, each well was washed once with PBS and stained with $1.0 \%$ crystal violet. Next, each well was washed three times with PBS, followed by solubilization with $33 \%$ acetic acid. The absorbance of the obtained solution was measured at $570 \mathrm{~nm}$, and the sessile biomass was presented as an $\mathrm{A}_{570}$ value.

\section{Effect of $\mathrm{pH}$ and Temperature on Endolysin Activity}

For the temperature stability assay of Lys109, the lytic activity was measured in reaction buffer at $25^{\circ} \mathrm{C}$ for $60 \mathrm{~min}$ after the enzyme was incubated at various temperatures $\left(4-65^{\circ} \mathrm{C}\right)$ for $30 \mathrm{~min}$. To study the effects of temperature on Lys109 enzymatic activity, 300 nM Lys109 was added into the target cell suspension, and the mixture was incubated at different temperatures $\left(4-65^{\circ} \mathrm{C}\right)$ for $60 \mathrm{~min}$. Then, the lytic activity was measured in reaction buffer at $25^{\circ} \mathrm{C}$. To test the effects of $\mathrm{pH}$ on the activity of Lys109, $300 \mathrm{nM}$ Lys109 was added to $S$. aureus CCARM 3090 cells suspended in the following buffers: $50 \mathrm{mM}$ sodium acetate ( $\mathrm{pH} 4.5$ and 5.4), $50 \mathrm{mM}$ Tris- $\mathrm{HCl}$ ( $\mathrm{pH} 6.5-8.0), 50 \mathrm{mM}$ glycine ( $\mathrm{pH} 9.0)$, and $50 \mathrm{mM}$ N-cyclohexyl-3-aminopropanesulfonic acid ( $\mathrm{pH} 10.0$ ).

\section{EGFP Fusion Protein Binding Assay}

The binding ability of EGFP_LysSA12 amidase plus CBD and EGFP_LysSA97 amidase plus CBD to $S$. aureus cells was evaluated as previously described (Loessner et al., 2002). Bacterial cells grown to the early exponential phase were harvested and resuspended in PBS. EGFP fusion proteins were incubated with the cells for $5 \mathrm{~min}$ at $25^{\circ} \mathrm{C}$. The mixture was washed twice with PBS to remove unbound protein and was moved to a 96-well plate to measure the fluorescence using a SpectraMax i3 multimode microplate reader (excitation at $485 \mathrm{~nm}$ and emission at $535 \mathrm{~nm}$ ). The $\mathrm{OD}_{600}$ of the cells was measured to normalize the fluorescence by calculating the whole-cell fluorescence per $\mathrm{OD}_{600}$.

\section{Antimicrobial Activity Assay in Food Samples}

The lytic activity of Lys109 against the MRSA CCARM 3090 strain was tested in commercial whole-fat pasteurized milk as previously described (Chang et al., 2017a). A milk sample was inoculated with exponentially growing MRSA CCARM 3090 cells (approximately $10^{5} \mathrm{CFU} / \mathrm{mL}$ ). Before the addition of Lys109 and LysSA12 at 0, 30, 300, 900, and $1500 \mathrm{nM}$, the milk samples were preincubated with the bacteria at $25^{\circ} \mathrm{C}$ for $1 \mathrm{~h}$ to allow the bacteria to adapt to the milk. Each milk sample was then incubated at $25^{\circ} \mathrm{C}$ for an additional hour. Viable bacterial cells $(\mathrm{CFU} / \mathrm{mL})$ were counted every $15 \mathrm{~min}$ after the addition of Lys109 and LysSA12 by plating each sample on a BPA plate and incubation at $37^{\circ} \mathrm{C}$ for $24 \mathrm{~h}$.

\section{Antimicrobial Activity Assay on Stainless Steel}

The lytic activity of Lys109 against the MRSA CCARM 3090 strain was tested on stainless steel as previously described (Cha et al., 2019). A stainless steel coupon with a size of $2 \times 2 \mathrm{~cm}^{3}$ was sterilized in an autoclave. Exponentially growing MRSA CCARM 3090 bacterial cells were harvested and resuspended in PBS to a final concentration of approximately $10^{5} \mathrm{CFU} / \mathrm{mL}$. Prepared bacterial cells were pipetted onto the stainless steel surface and dried for $1 \mathrm{~h}$ on a clean bench. Subsequently, the stainless steel samples were treated with Lys109 (0-100 nM) and left for $60 \mathrm{~min}$ at $25^{\circ} \mathrm{C}$. For the negative control, PBS was used instead of Lys109 solution. S. aureus cells were detached from the surface by agitation in PBST for 2 min with a benchtop vortex mixer at maximum speed. Cell suspensions were serially diluted and plated onto BPA plates and incubated at $37^{\circ} \mathrm{C}$ for $24 \mathrm{~h}$.

\section{Statistical Analysis}

GraphPad Prism (version 5.01) was used to conduct statistical analysis. One-way analysis of variance (ANOVA) followed by one-way Tukey's test for all pairwise comparisons (95\% confidence interval) was performed. The data are presented as the means with standard deviations. A $P$-value $<0.05$ was considered statistically significant. 


\section{RESULTS}

\section{Development of a Random Domain Swapping Method}

The overall scheme of random domain swapping method was presented in Figure 1A. The protocol was established based on a 96 well plate format for the rapid and efficient screening, taking advantage of a $S$. Typhimurium phage SPN1S lysis cassette composed of an endolysin and Rz/Rz1-like proteins (SPN1S lysRz) (Lim et al., 2012). SPN1S lysRz has been reported to cause host cell lysis and release viral progeny at the end of phage life cycle. To evaluate the cell lysis efficiency of SPN1S lysRz, the amount of EGFP protein released from lysed E. coli was measured. Fluorescence significantly increased after induction with arabinose (Figure 1B), indicating that SPN1S lysRz can cause the rapid lysis of $E$. coli cells from within, thereby releasing accumulated proteins in the cytosol. To identify an active chimeric endolysin from a large random library, the lytic efficacy of the released proteins was evaluated by their ability to form a clear zone on an agar plate overlaid with heat-killed $S$. aureus cells. As positive controls, four different $S$. aureus phage endolysins (LysSA11, LysSA97, LysSAP4, and LysSA12) were expressed in the presence of pBAD33_SPN1S lysRz. The clear zones were visualized on the agar plate depending on their activities, whereas clones without pBAD33_SPN1S lysRz did not show a clear zone (Figure 1C). These results suggest that SPN1S lysRz-induced lysis of $E$. coli allowed active chimeric endolysins to form clear zone on agar plates containing target bacteria and that this system can be used as a method for the successful screening of novel chimeric endolysins.

\section{Isolation of a Novel Endolysin Lys109}

The random library containing 480 clones were cotransformed into E. coli containing the SPN1S lysRz-harboring vector and

A
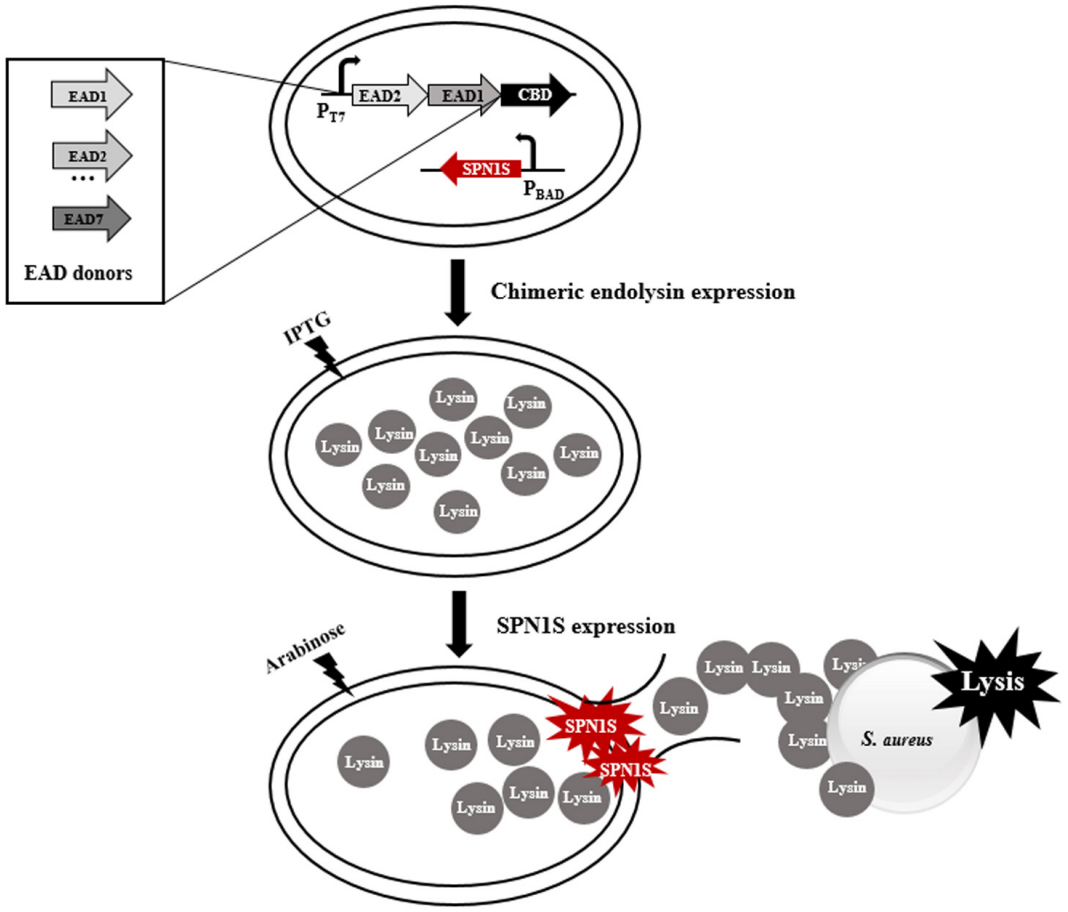

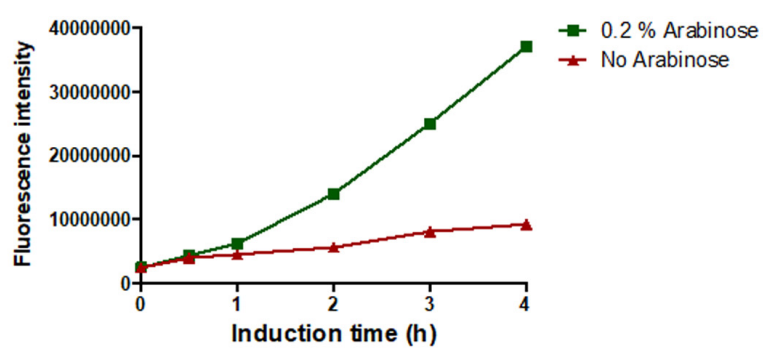

C

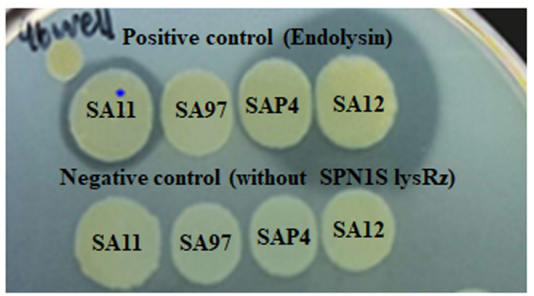

FIGURE 1 | Development of screening system on a 96-well microplate. (A) Scheme of the random domain screening method. (B) EGFP released from SPN1S-induced lysis in host $E$. coli cells. Cells without induction IPTG for EGFP expression and arabinose for SPN1S lysRz expression) were used as a control. (C) Clones for positive and negative controls were cultured with $0.2 \%$ arabinose overnight on the lawn of autoclaved S. aureus RN4220. 
applied to an agar plate containing $S$. aureus for screening. The clones displaying clear lysis zones were sequenced to determine the combination of the EADs (Supplementary Table S2). Most of the selected clones contained a CHAP domain from LysSA12 or LysSA11, which showed high staphylolytic activity (Chang et al., 2017a,b). In particular, LysSA12 CHAP domain-containing clones, which account for $68 \%$ of selected clones, showed a large and clear lysis zone. There was also a single clone containing a LysSAP4 CHAP domain. These results suggest that CHAP domain is necessary to degrade $S$. aureus cell wall peptidoglycan, and this is consistently observed in other chimeric endolysins to control S. aureus (Daniel et al., 2010; Schmelcher et al., 2012c; Yang et al., 2014). Among LysSA12 CHAP domaincontaining clones, five promising chimeric endolysins were

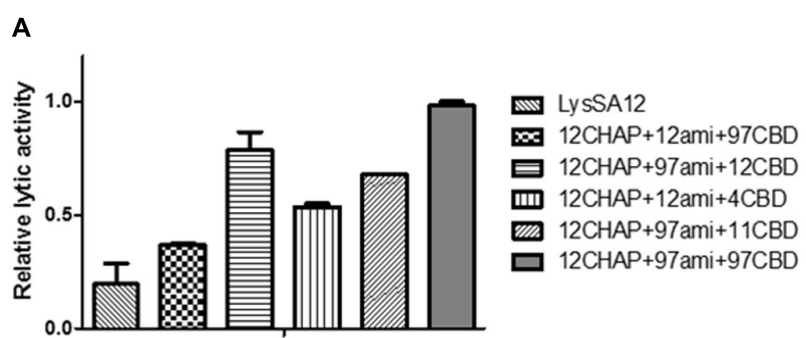

B

C

\begin{tabular}{|c|c|c|c|}
\hline Lys109 & SA12 CHAP & SA97 amidase & SA97 CBD \\
\hline LysSA12 & SA12 CHAP & SA12 amidase & SA12 CBD \\
\hline LysSA97 & SA97 CHAP & SA97 amidase & SA97CBD \\
\hline
\end{tabular}

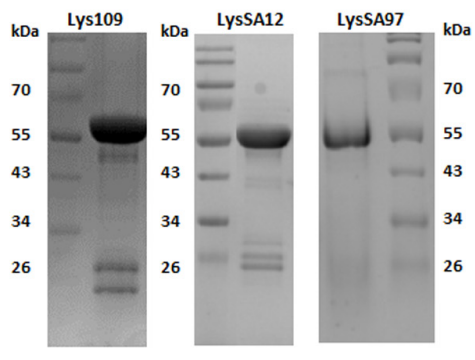

D

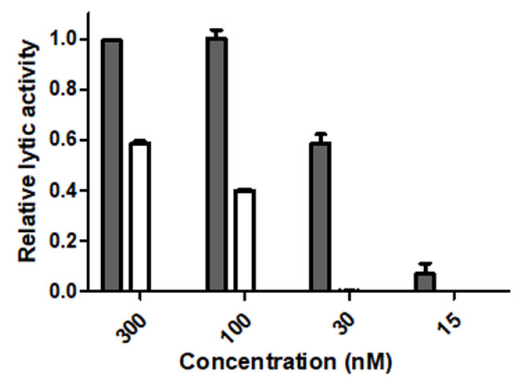

$\square$ Lys 109 $\square$ LysSA12 曰 LysSA97

E

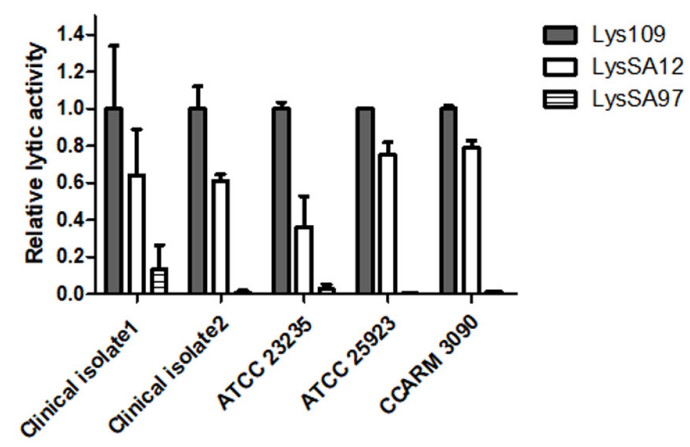

F

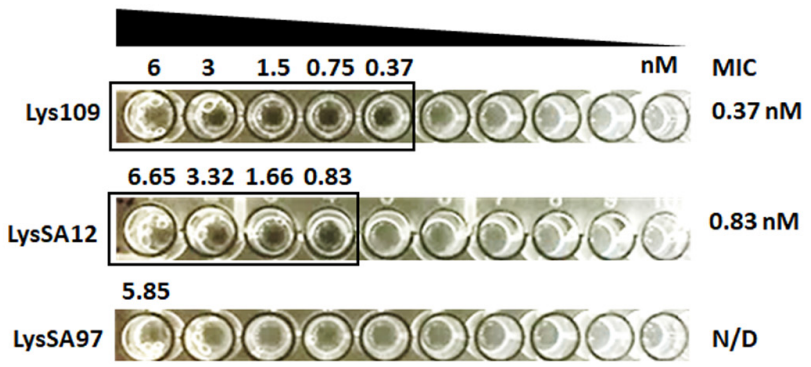

FIGURE 2 | Lytic activity comparison of Lys109 with parental endolysins. (A) Comparison of the relative lytic activity of four other chimeric endolysins and LysSA12 with Lys109 (300 nM each) against S. aureus CCARM 3090. (B) Schematic representation of Lys109, LysSA12 and LysSA97. (C) SDS-PAGE analysis of Lys109, LysSA12, and LysSA97. (D) Relative lytic activity of Lys109, LysSA12 and LysSA97 against S. aureus CCARM 3090 at different concentrations. (E) Relative lytic activity of Lys109, LysSA12 and LysSA97 (300 nM each) against various S. aureus strains. (F) MIC values of Lys109, LysSA12 and LysSA97 against S. aureus CCARM 3090. 
selected for the further comparative analysis (Figure 2A). All selected proteins were expressed in E. coli in soluble form and evaluated for their lytic activity against $S$. aureus. As a result, a chimeric endolysin consisting of a LysSA12 CHAP domain in the N-terminal region, a LysSA97 amidase domain at the central and a LysSA97 CBD in the C-terminal region showed the highest lytic activity among the five candidates, and was designated Lys109 (Figure 2B). BLAST analysis revealed that Lys109 has 78\% overall amino acid sequence identity with an amidase from S. aureus phage StauST398-1 (Van Der MeeMarquet et al., 2013) and 80\% with endolysins from $\Phi B 166$ and ФB236 S. aureus phage (Botka et al., 2015). Although the three endolysins showed high similarity with Lys109, they have not yet been studied, suggesting that further research on Lys109 will be meaningful. Moreover, LysSA12, a CHAP domain donor of Lys109, has high amino acid similarity with LysH5 of S. aureus phage vB_SauS_phiIPLA88 (98\% identity to LysSA12) and S. aureus $\Phi 11$ endolysin (96\% identity to LysSA12) (Sass and Bierbaum, 2007; García et al., 2010). These endolysins have a conserved catalytic triad (C32, H95, and Q112) in their CHAP domains (Supplementary Figure S1). Considering that the lytic activity of $S$. aureus endolysin depends mostly on a CHAP domain, we selected Lys109 to investigate the effect of domain replacement on the activity of the endolysin.

\section{Lytic Activity of Lys109 in Comparison With Its Parental Endolysins}

Lys109 and its parental endolysins, LysSA12 and LysSA97, were highly expressed as a soluble form in E. coli and were purified via Ni-NTA affinity chromatography. The predicted molecular weights of Lys109, LysSA12 and LysSA97 were approximately $54 \mathrm{kDa}$ and the proteins migrated as expected in SDS-PAGE gel (Figure 2C). The antibacterial activity of Lys109 was compared with those of LysSA12 and LysSA97 at various concentrations (Figure 2D). Lys109 exhibited clear cell lysis against S. aureus CCARM 3090, displaying at least 2-fold higher lytic activity than that of LysSA12 and LysSA97 at all tested concentrations. LysSA12 did not show lytic activity at concentrations below $30 \mathrm{nM}$ and LysSA97 barely exhibited staphylolytic activity at all tested concentrations. These results indicate the superiority of lytic activity of LysSA12 CHAP over LysSA97 CHAP despite their sequence similarity (44\% identity). Comparative analysis of the lytic activity of Lys109 with its parental endolysins against other S. aureus strains including clinical isolates and MRSA also showed an evident improvement in the lytic activity of Lys109 (Figure 2E). The MIC of Lys109 was compared with those of its parental endolysins. LysSA97, a donor for the amidase domain and CBD of Lys109, did not show inhibition of cell growth at the maximum concentrations available $(5.85 \mu \mathrm{M})$ (Figure 2F), leading us to exclude LysSA97 in the following experiments. LysSA12 inhibited the growth of S. aureus CCARM 3090 at concentration of $0.843 \mu \mathrm{M}$. The MIC of Lys109 was $0.375 \mu \mathrm{M}$, which was at least 2.25-fold lower than that of LysSA12, indicating that the antimicrobial activity of Lys109 was significantly improved compared to its parental endolysins.

\section{Biofilm Reduction Activity of Lys109}

The biofilm reduction efficacy of Lys109 against biofilms formed by S. aureus CCARM 3090 and S. aureus RN4220 was evaluated by a crystal violet staining-based assay (Figure 3). Lys109 showed biofilm reduction activity in a dose-dependent manner, and Lys109 appeared to have a higher biofilm reduction efficacy than LysSA12. When $300 \mathrm{nM}$ of endolysins were added to the biofilms, Lys 109 exhibited more than 3-fold enhanced efficacy in removing the biofilms compared to LysSA12. These results demonstrate that Lys109 has strong lytic activity against not only planktonic cells but also biofilms, which is an important contributing factor for many treatment failures (Otto, 2013).

\section{Temperature and pH Effects on the Enzymatic Activity of Lys109}

The thermostability of endolysins was determined (Supplementary Figure S2). Lys109 retained over 95\% of its activity after $1 \mathrm{~h}$ of incubation at 4 to $37^{\circ} \mathrm{C}$, and the lytic activity of Lys 109 started to decrease at $45^{\circ} \mathrm{C}$. Approximately $40 \%$ decrease in its hydrolytic activity was observed at $50^{\circ} \mathrm{C}$, and higher temperatures $\left(55\right.$ and $\left.65^{\circ} \mathrm{C}\right)$ caused complete inactivation of Lys109. LysSA12 showed similar pattern of thermal stability to that of Lys109, indicating that the structural stabilization by the fusion of LysSA12 CHAP to LysSA97 amidase domain plus CBD might not be the reason of the enzymatic improvement of Lys109. Next, the effects of temperature and $\mathrm{pH}$ on the lytic activity of Lys109 were evaluated to determine the optimum working conditions of Lys109 (Figures 4A,B). The maximal activity of Lys 109 was exhibited at $25-37^{\circ} \mathrm{C}$ and $\mathrm{pH}$ 6.5-9.0. The wide optimum $\mathrm{pH}$ range of Lys109 suggest that Lys109 can be used for a wide variety of applications, including foods associated with a high risk for $S$. aureus contamination, such as milk products (Marino et al., 2000) and disinfectants for hospital cleaning (Dancer, 2008).

\section{Antibacterial Spectrum of Lys109}

The antimicrobial activity of Lys109 was examined against staphylococcal strains other Gram-positive and Gram-negative bacteria with different amounts of endolysins, and was compared to that of LysSA12 (Table 1). Lys109 displayed effective lytic activity against all tested staphylococcal strains but not the other Gram-positive and Gram-negative bacteria tested. Although Lys109 has the same antibacterial spectrum as LysSA12, Lys109 showed higher lytic activity against most staphylococcal strains tested compared with LysSA12. These results demonstrated that Lys 109 retains the specificity of the original endolysin but has stronger antimicrobial properties.

\section{Efficacy of Lys109 Against S. aureus in Milk}

The comparative antibacterial activity of Lys109 and LysSA12 against a MRSA (S. aureus CCARM 3090) strain was examined at various concentrations in milk. Milk was chosen for the test because they have been frequently implicated in staphylococcal foodborne illnesses (Kadariya et al., 2014). In milk artificially contaminated with S. aureus, treatment with LysSA12 did not 
A
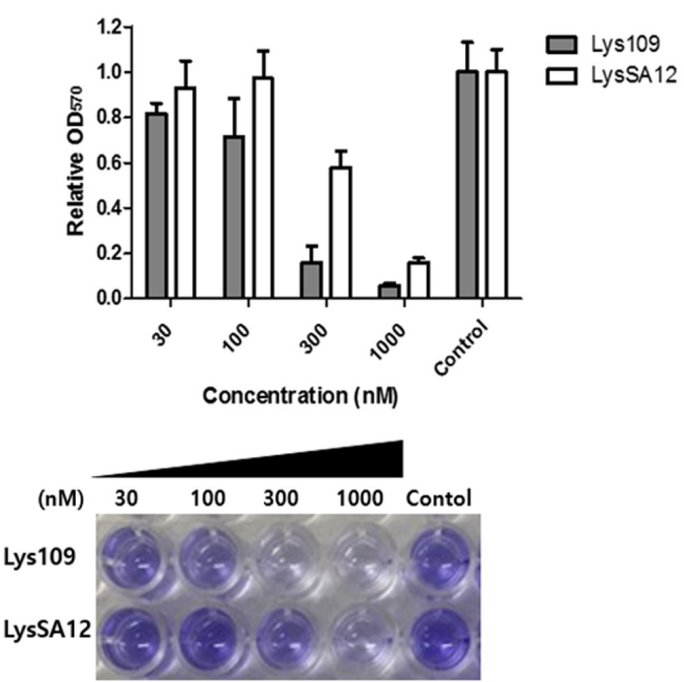

B S. aureus RN4220

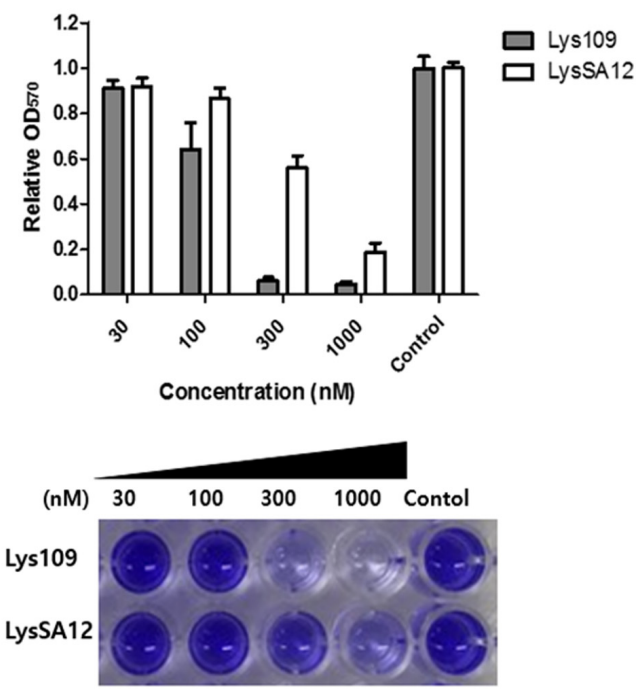

FIGURE 3 | Biofilm reduction activity of Lys109 and LysSA12. Biofilms formed by (A) S. aureus CCARM 3090 and (B) S. aureus RN4220 were treated with various concentrations of Lys109 and LysSA12 and visualized by staining biofilms with crystal violet. Dark staining indicates the biofilm that was maintained after treatment with the endolysin. Light or no staining indicates successful removal of the biofilm. Control indicates the sample treated with buffer without endolysins.

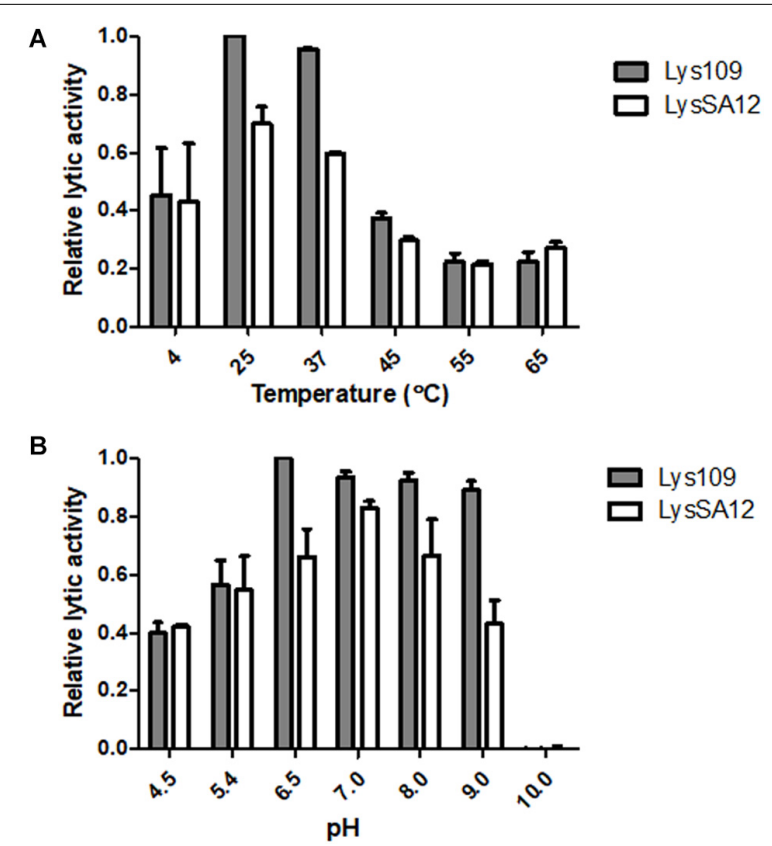

FIGURE 4 | The effects of temperature and $\mathrm{pH}$ on the lytic activity of Lys109 and LysSA12. The optimum (A) temperature and (B) pH of Lys109 and LysSA12 were examined by incubation of the enzymes (300 nM each) with target cells at different temperatures and in different $\mathrm{pH}$ buffers, respectively. The relative lytic activities were calculated using the activity of Lys109 assayed in a buffer at $\mathrm{pH} 6.5$ and $25^{\circ} \mathrm{C}$, which showed maximal activity.

show any reduction in CFU at all tested concentrations even though LysSA12 exerted lytic activity at $100 \mathrm{nM}$ in reaction buffer (Figure 5A), demonstrating the importance of in vivo experiments to evaluate the potential of a new antimicrobial. Similarly, LysH5, a LysSA12 homolog, could not kill bacteria cells in milk at $0.15 \mu \mathrm{M}$, and only 1-log reduction was observed with 0.8 MM LysH5 (Obeso et al., 2008; García et al., 2010). On the other hand, treatment with $300 \mathrm{nM}$ of Lys109 showed an apparent inhibitory effect on $S$. aureus within $15 \mathrm{~min}$ and resulted in a 2-log reduction of bacterial cells after $1 \mathrm{~h}$ (Figures 5B,C). In addition, the number of S. aureus in milk decreased below the detection limit with $900 \mathrm{nM}$ Lys109 within $45 \mathrm{~min}$ (Figure 5B), indicating improved antimicrobial activity of the chimeric endolysin compared to parental endolysins. These findings showed that Lys109 has high staphylolytic activity in a complex biomatrix as well as in buffer and suggest that Lys109 has great potential as an antimicrobial agent to control S. aureus from dairy products.

\section{Efficacy of Lys109 Against S. aureus on Stainless Steel}

Staphylococcus aureus is a common bacterium encountered in hospital-acquired and device-associated infections (Gwisai et al., 2017). Stainless steel is commonly used for medical devices, and previous reports revealed that MRSA can be viable and grow on it, leading to human infections (Michels et al., 2015; Craft et al., 2019). In this regard, the staphylolytic efficacy of Lys 109 was evaluated and compared with its parental endolysin, LysSA12, on stainless steel coupons artificially contaminated with $S$. aureus CCARM 3090. At 100 nM, treatment with Lys109 on the surface of a stainless steel coupon caused the number of bacterial cells to decrease below the detection limit, whereas its parental endolysin was less effective in bacterial cell killing, resulting in only a 1-log bacterial reduction after $1 \mathrm{~h}$ (Figure 6). These results suggest the possible use of Lys109 as a disinfectant in hospital settings. 


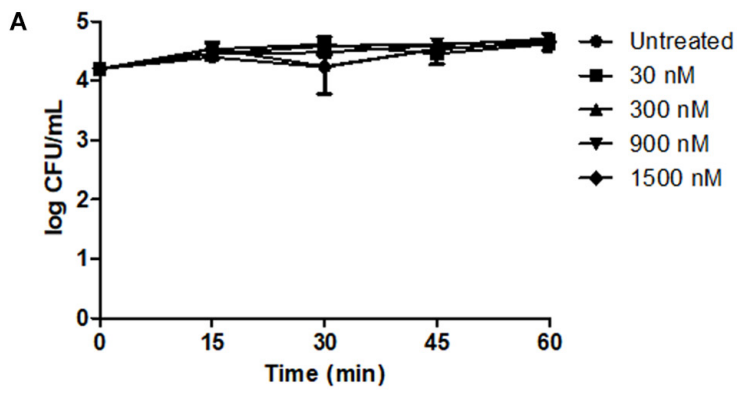

B

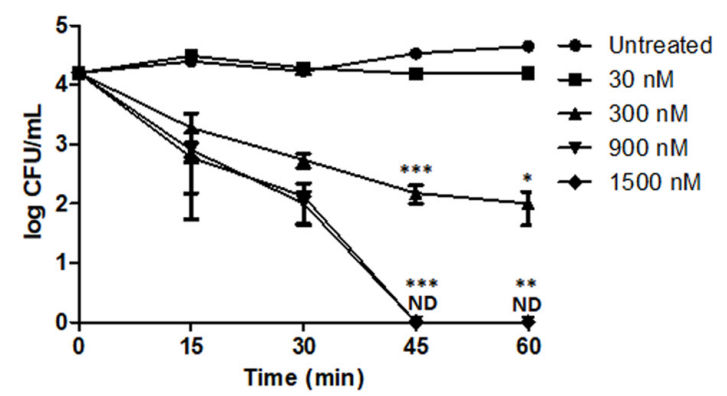

C

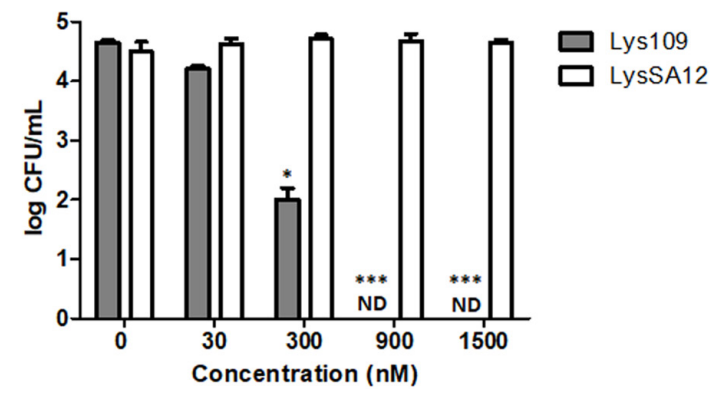

FIGURE 5 | Antibacterial activity of Lys109 and LysSA12 against S. aureus CCARM 3090 in milk. (A) LysSA12 and (B) Lys109 were added to milk artificially contaminated with S. aureus CCARM 3090 at different concentrations. Bacterial cells were counted every 15 min for $1 \mathrm{~h}$. (C) The number of $S$. aureus CCARM 3090 cells in milk was counted after $1 \mathrm{~h}$ of treatment with Lys 109 and LysSA12 at concentrations of $0 \mathrm{nM}$ (negative control), 30, 300, 900, and $1500 \mathrm{nM}$. ND, not detected. Asterisks indicate significant differences $\left.{ }^{\left({ }^{\star \star} P\right.} P<0.001,{ }^{\star \star} P<0.01,{ }^{*} P<0.05\right)$.

\section{DISCUSSION}

The emergence of multidrug-resistant $S$. aureus has called for novel therapeutic options beyond conventional antibiotics. Endolysins from staphylococcal phages have been proposed as promising alternatives to combat $S$. aureus due to their near species specificity and low probability of developing bacterial resistance (Kashani et al., 2018). In this regard, many researchers have tried to isolate $S$. aureus phages and to identify novel endolysins within the genomes of isolated phages. However, this approach is time-consuming and usually inefficient because staphylococcal endolysins, which are generally classified into five major groups according to their domain architectures (Chang and Ryu, 2017), have more than 90\% amino acid sequence identity within each group (Becker et al., 2009b).

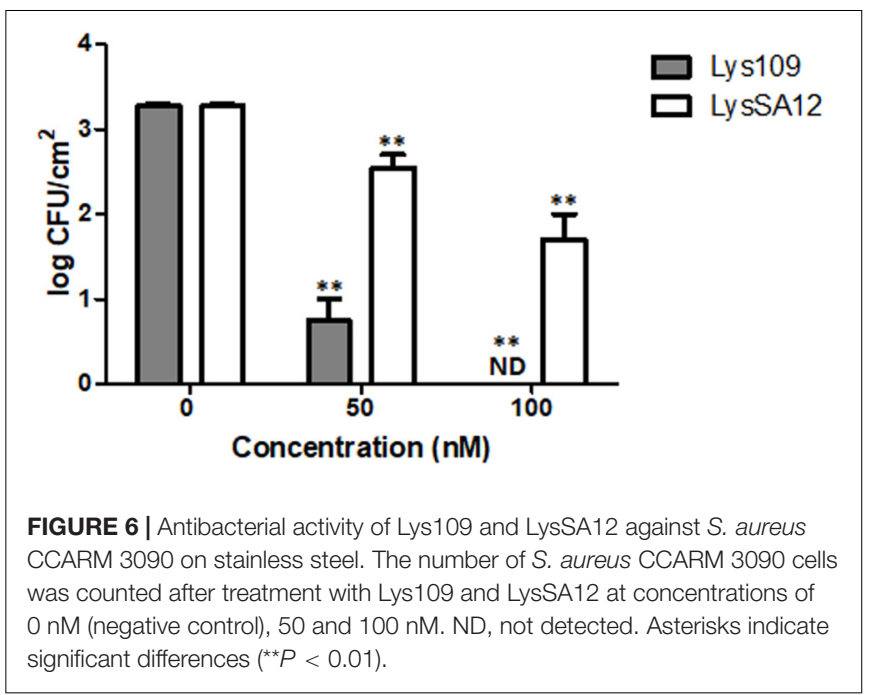

Besides, poor expression and low solubility levels have further aggravated the situation of getting highly active $S$. aureus endolysins (Manoharadas et al., 2009; Daniel et al., 2010; García et al., 2010). For these reasons, developing a novel S. aureus-targeting endolysin with desired properties is of both commercial and academic interest. In this study, we propose an endolysin engineering strategy based on the random domain swapping method to get highly active $S$. aureustargeting endolysins.

Our screening method involves several noteworthy features as follows. First, we utilized the lytic ability of $S$. Typhimurium phage SPN1S lysRz cassette to lyse E. coli cells from within to release expressed endolysins from the cytosol. The SPN1S lysRz cassette consists of an endolysin and two component spanins ( $\mathrm{Rz}$ and Rz1) which are reported to be essential for disrupting the outer membrane in this final step of host lysis (Rajaure et al., 2015). The co-expression of SPN1S lysRz resulted in strong cell lysis and we were rapidly able to screen highly active chimeric endolysins using 96-well plate format. Second, we constructed two different types of random domain libraries to increase the library diversity, one with variable number of EADs in a random orientation, and the other with only two EADs. A total 19 clones were selected from the libraries based on their strong lytic profiles on $S$. aureus lawns. Sequence analysis revealed that all 19 clones have one or two CHAP domains at the N-terminus and 13 out of the 19 selected clones harbored an amidase domain in the middle of the chimeric endolysins. Consistent with previous reports (Becker et al., 2009a; Schmelcher et al., 2012b; Son et al., 2018), our results indicate that the N-terminal CHAP domain is essential for lysis of $S$. aureus cells and that the amidase domain at the central region may enhance the overall lytic activity of endolysins. Third, the use of heat-killed $S$. aureus cells allowed us to distinguish clearly active chimeric endolysins among clones in the screening process. When E. coli and $S$. aureus were co-cultured, it gave rise to false positive clones, some of which showed clear zone on the lawn of $S$. aureus but produced insoluble or inactive form of proteins. Considering 
that E. coli grows faster than $S$. aureus in mixed culture (Fujikawa and Sakha, 2014), we speculate that E. coli might inhibit the growth of $S$. aureus, forming inhibition-like zone on the $S$. aureus lawn.

We found that this screening strategy was successful for developing a novel chimeric endolysin and Lys109, which showed the most effective staphylolytic activity, was selected from the random libraries. Lys109 was composed of a LysSA12 CHAP domain, a LysSA97 amidase domain, and a LysSA97 CBD. The efficacy of Lys109 to remove staphylococcal planktonic cells and biofilms was much stronger than that of its parental endolysins, LysSA12 and LysSA97. Then, how Lys109 has superior lytic activity to its parental endolysins? One possible reason is that the increased binding ability of the chimeric endolysin might have led to the improvement of antibacterial activity (Son et al., 2018). Indeed, LysSA97 amidase plus CBD displayed higher binding affinity to the target bacteria than LysSA12 amidase plus CBD (Supplementary Figure S3), suggesting that LysSA97 amidase plus CBD increases the lytic activity of LysSA12 CHAP by enhancing its accessibility to the target bacteria. Alternatively, the peptidoglycan fragment generated by the initial LysSA12 CHAP digestion could be more sensitive to the LysSA97 amidase domain plus CBD than the LysSA12 amidase plus CBD (Becker et al., 2009a). Further structural and biophysical studies of Lys109 will be needed to verify the exact molecular mechanism of enhanced lytic activity provided by domain swapping.

Lys109 showed much stronger antimicrobial activity than its parental endolysin, LysSA12 in milk. The treatment with 300 $\mathrm{nM}$ of Lys109 for $1 \mathrm{~h}$ showed 2-log reduction of bacterial cells in milk and $900 \mathrm{nM}$ of Lys109 was sufficient to reduce the staphylococcal cells to undetectable levels within $45 \mathrm{~min}$. Several other peptidoglycan hydrolases also have been examined for their antimicrobial activity in milk, but they generally showed low lytic activity in milk (Schmelcher et al., 2015; Verbree et al., 2018). LysH5, a LysSA12 homolog, could not kill bacterial cells in milk with $0.15 \mu \mathrm{M}$ and only a 1-log reduction was observed with $0.8 \mu \mathrm{M}$ LysH5 (Obeso et al., 2008; García et al., 2010). The antimicrobial activity of LysSA97, a donor for an amidase and a CBD of Lys109, was also marginal when $1.88 \mu \mathrm{M}$ of the protein was added in milk (Chang et al., 2017b). More recently, LysSA11 derived from S. aureus phage SA11 showed only a 1-log reduction of staphylococcal cell counts in milk when it was added at 2.25 $\mathrm{MM}$ for $1 \mathrm{~h}$ (Chang et al., 2017a). These results indicate the potential of Lys109 as antimicrobial additives for milk products. Besides, we observed that Lys 109 has strong antimicrobial activity on stainless steel. The result demonstrates that stainless steel, the material for medical devices, does not significantly affect the staphylolytic activity of Lys109 and that Lys109 can be possibly used as a disinfectant in clinical settings.

In summary, we proposed an effective screening method to find a novel chimeric endolysin with higher lytic activity and

\section{REFERENCES}

Andrews, J. M. (2001). Determination of minimum inhibitory concentrations. J. Antimicrob. Chemother. 48, 5-16. doi: 10.1093/jac/48.suppl_1.5 solubility through a random domain swapping of $S$. aureus endolysins. Lys109 was selected from random libraries and showed much stronger lytic activity against staphylococcal strains than its parental endolysins, LysSA12 and LysSA97. Moreover, Lys109 effectively removed staphylococcal biofilms and significantly reduced the number of $S$. aureus cells in milk and on the surface of stainless steel. These results suggest that the random domain swapping method can provide an opportunity for researchers to generate a novel and potent chimeric endolysin with minimal effort. Our strategy therefore holds considerable potential for medical and biotechnological applications to combat multidrug-resistant bacteria such as S. aureus.

\section{DATA AVAILABILITY STATEMENT}

The raw data supporting the conclusion of this article will be made available by the authors, without undue reservation.

\section{AUTHOR CONTRIBUTIONS}

BS and SR conceived and designed the experiments. BS performed the experiments and analyzed the data. YL participated in the experiments. BS and MK wrote the manuscript. SR revised the manuscript. All authors have read and accepted the final manuscript.

\section{FUNDING}

This work was supported by Basic Science Research Programs (2020R1A2B5B03094303) through the National Research Foundation of Korea (NRF) funded by the Ministry of Science, ICT and Future Planning and a grant (20162MFDS142) from Ministry of Food and Drug Safety in 2020.

\section{ACKNOWLEDGMENTS}

The content of this manuscript has been published in part as part of the thesis of BS (Son, 2019).

\section{SUPPLEMENTARY MATERIAL}

The Supplementary Material for this article can be found online at: https://www.frontiersin.org/articles/10.3389/fmicb. 2020.615887/full\#supplementary-material

Becker, S. C., Dong, S., Baker, J. R., Foster-Frey, J., Pritchard, D. G., and Donovan, D. M. (2009a). LysK CHAP endopeptidase domain is required for lysis of live staphylococcal cells. FEMS Microbiol. Lett. 294, 52-60. doi: 10.1111/j.15746968.2009.01541.x 
Becker, S. C., Foster-Frey, J., Stodola, A. J., Anacker, D., and Donovan, D. M. (2009b). Differentially conserved staphylococcal SH3b_5 cell wall binding domains confer increased staphylolytic and streptolytic activity to a streptococcal prophage endolysin domain. Gene 443, 32-41. doi: 10.1016/j. gene.2009.04.023

Borysowski, J., Weber-Dąbrowska, B., and Górski, A. (2006). Bacteriophage endolysins as a novel class of antibacterial agents. Exp. Biol. Med. 231, 366-377. doi: 10.1177/153537020623100402

Botka, T., Rùžièková, V., Koneèná, H., Pantùèek, R., Rychlík, I., Zdráhal, Z., et al. (2015). Complete genome analysis of two new bacteriophages isolated from impetigo strains of Staphylococcus aureus. Virus Genes 51, 122-131. doi: 10. 1007/s11262-015-1223-8

Cha, Y., Son, B., and Ryu, S. (2019). Effective removal of staphylococcal biofilms on various food contact surfaces by Staphylococcus aureus phage endolysin LysCSA13. Food Microbiol. 84:103245. doi: 10.1016/j.fm.2019.103245

Chambers, H. F., and Deleo, F. R. (2009). Waves of resistance: Staphylococcus aureus in the antibiotic era. Nat. Rev. Microbiol. 7:629. doi: 10.1038/ nrmicro2200

Chang, Y., Kim, M., and Ryu, S. (2017a). Characterization of a novel endolysin LysSA11 and its utility as a potent biocontrol agent against Staphylococcus aureus on food and utensils. Food Microbiol. 68, 112-120. doi: 10.1016/j.fm. 2017.07.004

Chang, Y., and Ryu, S. (2017). Characterization of a novel cell wall binding domain-containing Staphylococcus aureus endolysin LysSA97. Appl. Microbiol. Biotechnol. 101, 147-158. doi: 10.1007/s00253-016-7747-6

Chang, Y., Yoon, H., Kang, D.-H., Chang, P.-S., and Ryu, S. (2017b). Endolysin LysSA97 is synergistic with carvacrol in controlling Staphylococcus aureus in foods. Int. J. Food Microbiol. 244, 19-26. doi: 10.1016/j.ijfoodmicro.2016.12.007

Craft, K. M., Nguyen, J. M., Berg, L. J., and Townsend, S. D. (2019). Methicillinresistant Staphylococcus aureus (MRSA): antibiotic-resistance and the biofilm phenotype. MedChem Comm 10, 1231-1241. doi: 10.1039/c9md00044e

Dancer, S. J. (2008). Importance of the environment in meticillin-resistant Staphylococcus aureus acquisition: the case for hospital cleaning. Lancet Infect. Dis. 8, 101-113. doi: 10.1016/s1473-3099(07)70241-4

Daniel, A., Euler, C., Collin, M., Chahales, P., Gorelick, K. J., and Fischetti, V. A. (2010). Synergism between a novel chimeric lysin and oxacillin protects against infection by methicillin-resistant Staphylococcus aureus. Antimicrob. Agents Chemother. 54, 1603-1612. doi: 10.1128/aac.01625-09

De Lencastre, H., Oliveira, D., and Tomasz, A. (2007). Antibiotic resistant Staphylococcus aureus: a paradigm of adaptive power. Curr. Opin. Microbiol. 10, 428-435. doi: 10.1016/j.mib.2007.08.003

Diaz, E., Lopez, R., and Garcia, J. L. (1990). Chimeric phage-bacterial enzymes: a clue to the modular evolution of genes. Proc. Natl. Acad. Sci. 87, 8125-8129. doi: 10.1073/pnas.87.20.8125

Fenton, M., Casey, P. G., Hill, C., Gahan, C. G., Mcauliffe, O., O'mahony, J., et al. (2010). The truncated phage lysin CHAPk eliminates Staphylococcus aureus in the nares of mice. Bioeng. Bugs 1, 404-407. doi: 10.4161/bbug.1.6.13422

Fernandes, S., Proença, D., Cantante, C., Silva, F. A., Leandro, C., Lourenço, S., et al. (2012). Novel chimerical endolysins with broad antimicrobial activity against methicillin-resistant Staphylococcus aureus. Microb. Drug Resist. 18, 333-343. doi: $10.1089 / \mathrm{mdr} .2012 .0025$

Fischetti, V. A. (2008). Bacteriophage lysins as effective antibacterials. Curr. Opin. Microbiol. 11, 393-400. doi: 10.1016/j.mib.2008.09.012

Foster, T. J. (2004). The Staphylococcus aureus "superbug". J. Clin. Invest. 114, 1693-1696. doi: 10.1172/jci200423825

Fujikawa, H., and Sakha, M. Z. (2014). Prediction of competitive microbial growth in mixed culture at dynamic temperature patterns. Biocontrol. Sci. 19, 121-127. doi: 10.4265/bio.19.121

García, P., Martínez, B., Rodríguez, L., and Rodríguez, A. (2010). Synergy between the phage endolysin LysH5 and nisin to kill Staphylococcus aureus in pasteurized milk. Int. J. Food Microbiol. 141, 151-155. doi: 10.1016/j. ijfoodmicro.2010.04.029

Gu, J., Xu, W., Lei, L., Huang, J., Feng, X., Sun, C., et al. (2011). LysGH15, a novel bacteriophage lysin, protects a murine bacteremia model efficiently against lethal methicillin-resistant Staphylococcus aureus infection. J. Clin. Microbiol. 49, 111-117. doi: 10.1128/jcm.01144- 10

Gwisai, T., Hollingsworth, N. R., Cowles, S., Tharmalingam, N., Mylonakis, E., Fuchs, B. B., et al. (2017). Repurposing niclosamide as a versatile antimicrobial surface coating against device-associated, hospital-acquired bacterial infections. Biomed. Mater. 12:045010. doi: 10.1088/1748-605x/aa7105

Idelevich, E. A., Von Eiff, C., Friedrich, A. W., Iannelli, D., Xia, G., Peters, G., et al. (2011). In vitro activity against Staphylococcus aureus of a novel antimicrobial agent, PRF-119, a recombinant chimeric bacteriophage endolysin. Antimicrob. Agents Chemother. 55, 4416-4419. doi: 10.1128/aac.00217-11

Kadariya, J., Smith, T. C., and Thapaliya, D. (2014). Staphylococcus aureus and staphylococcal food-borne disease: an ongoing challenge in public health. BioMed Res. Int. 2014:827965.

Kashani, H. H., Schmelcher, M., Sabzalipoor, H., Hosseini, E. S., and Moniri, R. (2018). Recombinant endolysins as potential therapeutics against antibioticresistant Staphylococcus aureus: current status of research and novel delivery strategies. Clin. Microbiol. Rev. 31:e00071-17.

Kerr, D. E., Plaut, K., Bramley, A. J., Williamson, C. M., Lax, A. J., Moore, K., et al. (2001). Lysostaphin expression in mammary glands confers protection against staphylococcal infection in transgenic mice. Nat. Biotechnol. 19, 66-70. doi: $10.1038 / 83540$

Kong, M., and Ryu, S. (2015). Bacteriophage PBC1 and its endolysin as an antimicrobial agent against Bacillus cereus. Appl. Environ. Microbiol. 81, 2274 2283. doi: 10.1128/aem.03485-14

Lewis, K. (2001). Riddle of biofilm resistance. Antimicrob. Agents Chemother. 45, 999-1007. doi: 10.1128/aac.45.4.999-1007.2001

Lim, J.-A., Shin, H., Kang, D.-H., and Ryu, S. (2012). Characterization of endolysin from a Salmonella Typhimurium-infecting bacteriophage SPN1S. Res. Microbiol. 163, 233-241. doi: 10.1016/j.resmic.2012.01.002

Loessner, M. J. (2005). Bacteriophage endolysins-current state of research and applications. Curr. Opin. Microbiol. 8, 480-487. doi: 10.1016/j.mib.2005. 06.002

Loessner, M. J., Kramer, K., Ebel, F., and Scherer, S. (2002). C-terminal domains of Listeria monocytogenes bacteriophage murein hydrolases determine specific recognition and high-affinity binding to bacterial cell wall carbohydrates. Mol. Microbiol. 44, 335-349. doi: 10.1046/j.1365-2958.2002. 02889.x

Lowy, F. D. (1998). Staphylococcus aureus infections. New Engl. J. Med. 339, $520-532$.

Manoharadas, S., Witte, A., and Bläsi, U. (2009). Antimicrobial activity of a chimeric enzybiotic towards Staphylococcus aureus. J. Biotechnol. 139, 118-123. doi: 10.1016/j.jbiotec.2008.09.003

Marino, T. G., West, L. A., Liewehr, F. R., Mailhot, J. M., Buxton, T. B., Runner, R. R., et al. (2000). Determination of periodontal ligament cell viability in long shelf-life milk. J. Endodont. 26, 699-702. doi: 10.1097/00004770-20001200000005

Michels, H. T., Keevil, C. W., Salgado, C. D., and Schmidt, M. G. (2015). From laboratory research to a clinical trial: copper alloy surfaces kill bacteria and reduce hospital-acquired infections. Health Environ. Res. Design J. 9, 64-79. doi: 10.1177/1937586715592650

Obeso, J. M., Martínez, B., Rodríguez, A., and García, P. (2008). Lytic activity of the recombinant staphylococcal bacteriophage $\Phi \mathrm{H} 5$ endolysin active against Staphylococcus aureus in milk. Int. J. Food Microbiol. 128, 212-218. doi: 10. 1016/j.ijfoodmicro.2008.08.010

Oliveira, H., Melo, L. D., Santos, S. B., Nóbrega, F. L., Ferreira, E. C., Cerca, N., et al. (2013). Molecular aspects and comparative genomics of bacteriophage endolysins. J. Virol. 87, 3277-3282.

Otto, M. (2012). MRSA virulence and spread. Cell. Microbiol. 14, 1513-1521. doi: $10.1111 /$ j.1462-5822.2012.01832.x

Otto, M. (2013). Staphylococcal infections: mechanisms of biofilm maturation and detachment as critical determinants of pathogenicity. Annu. Rev. Med. 64, 175-188. doi: 10.1146/annurev-med-042711-140023

Rajaure, M., Berry, J., Kongari, R., Cahill, J., and Young, R. (2015). Membrane fusion during phage lysis. Proc. Natl. Acad. Sci. U.S.A. 112, 5497-5502. doi: 10.1073/pnas.1420588112

Rashel, M., Uchiyama, J., Ujihara, T., Uehara, Y., Kuramoto, S., Sugihara, S., et al. (2007). Efficient elimination of multidrug-resistant Staphylococcus aureus by cloned lysin derived from bacteriophage ФMR11. J. Infect. Dis. 196, 1237-1247. doi: $10.1086 / 521305$

Sass, P., and Bierbaum, G. (2007). Lytic activity of recombinant bacteriophage $\varphi 11$ and $\varphi 12$ endolysins on whole cells and biofilms of Staphylococcus aureus. Appl. Environ. Microbiol. 73, 347-352. doi: 10.1128/aem.01616-06 
Schmelcher, M., Donovan, D. M., and Loessner, M. J. (2012a). Bacteriophage endolysins as novel antimicrobials. Fut. Microbiol. 7, 1147-1171. doi: 10.2217/ fmb. 12.97

Schmelcher, M., Korobova, O., Schischkova, N., Kiseleva, N., Kopylov, P., Pryamchuk, S., et al. (2012b). Staphylococcus haemolyticus prophage ФSH2 endolysin relies on cysteine, histidine-dependent amidohydrolases/peptidases activity for lysis 'from without'. J. Biotechnol. 162, 289-298. doi: 10.1016/j. jbiotec.2012.09.010

Schmelcher, M., Powell, A. M., Becker, S. C., Camp, M. J., and Donovan, D. M. (2012c). Chimeric phage lysins act synergistically with lysostaphin to kill mastitis-causing Staphylococcus aureus in murine mammary glands. Appl. Environ. Microbiol. 78, 2297-2305. doi: 10.1128/aem.07 050-11

Schmelcher, M., Shabarova, T., Eugster, M. R., Eichenseher, F., Tchang, V. S., Banz, M., et al. (2010). Rapid multiplex detection and differentiation of Listeria cells by use of fluorescent phage endolysin cell wall binding domains. Appl. Environ. Microbiol. 76, 5745-5756. doi: 10.1128/aem.00 801-10

Schmelcher, M., Shen, Y., Nelson, D. C., Eugster, M. R., Eichenseher, F., Hanke, D. C., et al. (2015). Evolutionarily distinct bacteriophage endolysins featuring conserved peptidoglycan cleavage sites protect mice from MRSA infection. J. Antimicrob. Chemother. 70, 1453-1465. doi: 10.1093/jac/dku552

Schmelcher, M., Tchang, V. S., and Loessner, M. J. (2011). Domain shuffling and module engineering of Listeria phage endolysins for enhanced lytic activity and binding affinity. Microb. Biotechnol. 4, 651-662. doi: 10.1111/j.1751-7915.2011. 00263.x

Son, B. (2019). Engineering of Bacteriophage Endolysins and Their Applications to Control and Detect Foodborne Pathogens. PhD Thesis. Seoul National University, Seoul.

Son, B., Kong, M., and Ryu, S. (2018). The auxiliary role of the amidase domain in cell wall binding and exolytic activity of staphylococcal phage endolysins. Viruses 10:284. doi: 10.3390/v10060284

Son, B., Yun, J., Lim, J.-A., Shin, H., Heu, S., and Ryu, S. (2012). Characterization of LysB4, an endolysin from the Bacillus cereus-infecting bacteriophage B4. BMC Microbiol. 12:33. doi: 10.1186/1471-2180-12-33
Swift, S. M., Seal, B. S., Garrish, J. K., Oakley, B. B., Hiett, K., Yeh, H.-Y., et al. (2015). A thermophilic phage endolysin fusion to a Clostridium perfringensspecific cell wall binding domain creates an anti-Clostridium antimicrobial with improved thermostability. Viruses 7, 3019-3034. doi: 10.3390/v706 2758

Van Der Mee-Marquet, N., Corvaglia, A.-R., Valentin, A.-S., Hernandez, D., Bertrand, X., Girard, M., et al. (2013). Analysis of prophages harbored by the human-adapted subpopulation of Staphylococcus aureus CC398. Infect. Genet. Evol. 18, 299-308. doi: 10.1016/j.meegid.2013.06.009

Verbree, C. T., Dätwyler, S. M., Meile, S., Eichenseher, F., Donovan, D. M., Loessner, M. J., et al. (2018). Corrected and republished from: Identification of peptidoglycan hydrolase constructs with synergistic staphylolytic activity in cow's milk. Appl. Environ. Microbiol. 84, e002134-17.

Wu, J. A., Kusuma, C., Mond, J. J., and Kokai-Kun, J. F. (2003). Lysostaphin disrupts Staphylococcus aureus and Staphylococcus epidermidis biofilms on artificial surfaces. Antimicrob. Agents Chemother. 47, 3407-3414. doi: 10.1128/ aac.47.11.3407-3414.2003

Yang, H., Linden, S. B., Wang, J., Yu, J., Nelson, D. C., and Wei, H. (2015). A chimeolysin with extended-spectrum streptococcal host range found by an induced lysis-based rapid screening method. Sci. Rep. 5:17257.

Yang, H., Zhang, Y., Yu, J., Huang, Y., Zhang, X.-E., and Wei, H. (2014). Novel chimeric lysin with high-level antimicrobial activity against methicillinresistant Staphylococcus aureus in vitro and in vivo. Antimicrob. Agents Chemother. 58, 536-542. doi: 10.1128/aac.01793-13

Conflict of Interest: The authors declare that the research was conducted in the absence of any commercial or financial relationships that could be construed as a potential conflict of interest.

Copyright (c) 2021 Son, Kong, Lee and Ryu. This is an open-access article distributed under the terms of the Creative Commons Attribution License (CC BY). The use, distribution or reproduction in other forums is permitted, provided the original author(s) and the copyright owner(s) are credited and that the original publication in this journal is cited, in accordance with accepted academic practice. No use, distribution or reproduction is permitted which does not comply with these terms. 